\title{
POINTS DE VUE EN CONFRONTATION DANS LES ANTIMÉTABOLES PLUS ET MOINS
}

\author{
Alain Rabatel \\ Armand Colin I Langue française
}

$2008 / 4-n^{\circ} 160$

pages 21 à 36

ISSN 0023-8368

Article disponible en ligne à l'adresse:

http://www.cairn.info/revue-langue-francaise-2008-4-page-21.htm

Pour citer cet article :

Rabatel Alain, «Points de vue en confrontation dans les antimétaboles PLUS et MOINS »,

Langue française, 2008/4 n 160, p. 21-36. DOI : 10.3917/ff.160.0021

Distribution électronique Cairn.info pour Armand Colin.

(c) Armand Colin. Tous droits réservés pour tous pays.

La reproduction ou représentation de cet article, notamment par photocopie, n'est autorisée que dans les limites des conditions générales d'utilisation du site ou, le cas échéant, des conditions générales de la licence souscrite par votre établissement. Toute autre reproduction ou représentation, en tout ou partie, sous quelque forme et de quelque manière que ce soit, est interdite sauf accord préalable et écrit de l'éditeur, en dehors des cas prévus par la législation en vigueur en France. II est précisé que son stockage dans une base de données est également interdit. 


\section{Points de vue en confrontation dans les antimétaboles PLUS et MoINS}

Ce travail porte sur l'antimétabole. Toutefois, compte tenu de la complexité de la figure et de la difficulté à la circonscrire - ce dont témoignent les flottements autour de sa définition -, on élargira dans la première partie la présentation de l'antimétabole aux autres figures dont elle est rapprochée. Ce tour d'horizon permettra d'avancer dans la compréhension de la figure et de dégager, dans la deuxième partie, deux grandes classes d'antimétabole, notées antimétaboles PLUS $(\mathscr{A}+)$ et antimétabole MOINS $(\mathscr{A}-)$, selon que les points de vue contenus dans les propositions inversées se conjuguent ou s'excluent. Dans la troisième partie, on verra que cette distinction rejaillit sur les diverses figures pragmatiques d'énonciateur qui émergent en discours, en tension entre la saisie du même et de l'autre.

\section{ETAT DES LIEUX}

L'antimétabole, parce qu'elle oppose des termes inversés, diffère de l'antithèse et du chiasme et est comparée à la réversion.

\section{I.I. Chiasme}

Le chiasme est défini comme une "antithèse dont on dispose les termes en miroir » (Reboul 1991 : 223). Il se caractérise par une opposition fondée sur une inversion (Reboul 1991: 134, Aquien 1993: 82s) et une double opération complète d'adjonction et de suppression (Dubois et alii $1970: 78$ ). Trois critères différencient le chiasme d'avec l'antimétabole ${ }^{1}$ : 
a) Le chiasme (1) - comme l'antithèse (2) - se déploie dans une seule prédication :

(1) Le monde des affaires ne doit pas diriger les affaires du monde. (B. Thibault, Libération, 1-3-2007) ${ }^{2}$

(2) Monsieur Sarkozy est adepte des petites carottes et des gros bâtons. (B. Thibault, France Inter, 11-12-2006)

Certes, chiasmes et antithèses peuvent opposer deux prédications, comme en (3) et (4), respectivement,

(3) C'est pourquoi, malgré les menaces et les tentatives de freins, José a persévéré. Bové ose, osons Bové! (Y. Youlontas, site www.unisavecbove.com).

(4) Mon projet c'est vous! Son projet, c'est lui! (S. Royal, en meeting, Lyon),

mais leur présence dans une seule prédication les différencie, sur un plan syntactico-énonciatif, de l'antimétabole.

b) Il repose sur des éléments inversés qui ne sont pas nécessairement répétés terme à terme. C'est ce qui distingue la structure métalinguistique $N+\operatorname{Adj} /$ / Adj $+\mathrm{N}$ du chiasme grammatical dans «valse mélancolique et langoureux vertige!» (Baudelaire, apud Aquien 1993: 82), de l'antimétabole dans (« Je ne prétends pas justifier ma vie par mes livres, non plus que mes livres par ma vie ", Bernanos, apud Dupriez : 53), où se répète une même paire de mots en ordre inverse.

c) Il n'est pas nécessairement une figure syntaxique ${ }^{3}$, à la différence de l'antimétabole. Aquien 1993: 82s répertorie, outre le chiasme "grammatical » (ie syntaxique), le chiasme phonétique ("secouant dans mes yeux// leurs feux diamantés » : [ã ø//ø ã]), le chiasme sémantique ("ô fangeuse grandeur!// Sublime ignominie! [- +//+ -]), le chiasme rythmique (« De l'une il bénissait,// il maudissait de l'autre» [2 4//4 2]). À quoi s'ajoute le chiasme mélodique, avec inversion des courbes montante puis descendante (Morier 1998 : 205).

Bref, le chiasme (inversion sans répétition) est une figure moins contrainte que l'antimétabole mais davantage que l'antithèse qui oppose les termes ou les notions sans les inverser ni les répéter. C'est pourquoi (5) n'est pas une antimétabole stricto sensu : le terme «faible » est certes répété en fin de prédication et en tête de la suivante (anadiplose), mais dans les deux cas, Sarkozy est en position thématique : l'absence de structure inversive complète explique qu'il serait abusif de parler ici d'antimétabole, il s'agit d'une antithèse doublée d'une anadiplose :

(5) Au sujet de Sarkozy, j'ai entendu une bonne formule, l'autre fois, qui le résume bien, lui qui encourage les milliardaires à quitter la France : «Dur avec les faibles, et faible avec les forts ». ("La chronique d'Evariste», in ResPUBLICA n $\left.{ }^{\circ} 499,30-12-2006\right)$

\footnotetext{
2. Nombre de nos exemples ont été rassemblés lors de la campagne des présidentielles. Le faible rendement de la collecte nous a contraint à intégrer d'autres exemples et à limiter l'analyse pragmatique contextuelle des antimétaboles inscrites dans un genre et une scène d'énonciation spécifiques.

3. Contrairement au classement du chiasme parmi le sous-ensemble des répétitions syntaxiques chez Frédéric 1985.

4. Le "chiasme sonore », phonétique, diffère de l'antimétathèse, reposant sur une inversion graphique : « le fiat et le fait » (Claudel, apud Dupriez 1980 : 54).
} 


\section{I.2. Antimétabole et réversion}

L'inversion syntaxique, caractéristique de l'antimétabole, exige la répétition de mots dans un ordre inverse et dans des propositions successives ${ }^{5}$, comme dans la réversion (Dupriez, Molinié, Robrieux), les deux figures étant rapprochées de l'épanadiplose, c'est-à-dire deux propositions dont la première commence par un mot qui se trouve à la fin de la seconde : cf. "Rien ne me verra plus, je ne verrai plus rien » Hugo, apud Robrieux 1993 : 81-82. On peut en effet définir l'antimétabole et la réversion comme des figures formées d'une épanadiplose et d'une anadiplose (Cf. Wikipédia). Ainsi définies, renvoyant à une même réalité syntaxique, antimétabole et réversion sont synonymes.

Toutefois, toutes les inversions doublées d'une répétition ne s'analysent pas comme antimétabole. "Vide et amour, amour et vide " (Thériault, apud Dupriez 1980: 54) n'est pas caractérisé comme antimétabole, en l'absence de lien de subordination, qui rend impossible le changement de hiérarchisation et donc le changement de sens qui en découle. D'aucuns nomment "fausse antimétabole» ces répétitions inversives simplement coordonnées: le site < http://www.cafe.umontreal.ca/cle/cases/c2137.html> donne comme exemple «bousculade et querelle, querelle et bousculade». Certes, l'absence de subordonnant n'empêche pas un processus de hiérarchisation basé sur la logique du post hoc ergo propter hoc, faisant du premier terme une cause du procès dénoté par le second: dans un cas la bousculade provoque une querelle, dans le second, la querelle entraîne une bousculade. Néanmoins, l'inversion ne modifie pas le sens des termes: les contours sémantiques de "vide», "amour», " querelle », «bousculade » restent identiques.

On pourrait considérer comme fausses antimétaboles les énoncés suivants, plus ou moins proverbiaux :

(6) Bonnet blanc, blanc bonnet.

(6') Blanc bonnet, bonnet blanc.

(7) Bonnet blanc et blanc bonnet ${ }^{6}$.

(7') Blanc bonnet et bonnet blanc.

En utilisant cette formule pour renvoyer dos-à-dos les candidats du deuxième tour de l'élection présidentielle de 1969 (G. Pompidou et A. Poher), le communiste J. Duclos signifiait qu'à ses yeux, leurs différences partidaires (droite ou centre droit) étaient secondaires par rapport à leur acceptation du capitalisme. La figure indique une convergence fondamentale l'emportant sur des divergences superficielles, comme en (8), où J.-M. Le Pen réunit dans sa

5. Antimetabole is the repetition of words in successive clauses, but in reverse grammatical order. It is similar to chiasmus, although chiasmus does not use repetition of the same words or phrases. Ex : Ask not what your country can do for you ; ask what you can do for your country. (J.F. Kennedy) < http:/ / www.answers.com/topic/antimetabole >

6. NB : l'ordre Adj $+\mathrm{N} / \mathrm{N}+$ Adj n'est pas indifférent, si l'adjectif change de signification selon sa place. Cf. «Ce n'était pas seulement un homme brave, que celui-là [Jean Ouiller], c'était encore un brave homme. » (Dumas, Les Louves de Machecoul, Alterédit, 2007 : 33). Dans ce cas, les différences sont grammaticalisées. 
critique les candidats de droite et de gauche accusés de brader les intérêts de la France :

(8) Madame Royal et Monsieur Sarkozy, c'est bonnet rose et rose bonnet. (J.M. Le Pen, 1-5-2007)

L'ordre des prédications importe peu, puisque l'effet visé par ces antimétaboles est de mettre en relief le caractère factice de l'ordre des référents, des dénominations et qualifications, puisque tout revient en définitive au même, d'un méta point de vue. C'est pourquoi il est possible d'y incrémenter une conjonction de coordination marquant une alternative de type conjonctif (ou), éventuellement renforcé par un énoncé modalisant tel que disons voir, peu importe l'ordre, on dira dans cet ordre ou dans un autre, on dira indifféremment:

(9) Blanc bonnet (ou), peu importe, bonnet blanc.

(10) On dira indifféremment, bonnet blanc (ou) blanc bonnet.

Si la distinction entre fausse antimétabole et antimétabole paraît solide, celle entre antimétabole et réversion l'est moins. Fontanier établit un "distinguo subtil » entre antimétabole et réversion, au motif que, dans celle-ci, le sens des termes est différent, alors qu'il reste stable dans celle-là. Ainsi le sens ne varierait pas dans l'antimétabole suivante :

(11) Quand nous avons été lui porter sa pitance, Pinguet et moi, c'est-à-dire moi et Pinguet, il fondait en larmes, que l'on eût dit que ses deux yeux avaient deux robinets. (Dumas, Les Louves de Machecoul, Alterédit, 2007 : 486)

La réversion " fait revenir sur eux-mêmes, avec un sens différent, et souvent contraire, tous les mots, au moins les plus essentiels, d'une proposition » (Fontanier 1968: 381) :

(12) Ce ne sont pas les places qui honorent les hommes, mais les hommes qui honorent les places. (Agésilas, apud Fontanier, ibid.).

« Place » et « homme » sont d'abord pris en un sens négatif (" place » = sinécure ; homme $=$ personnage avide d'honneurs), ensuite en un sens positif : "place " = devoirs de la charge à accomplir au mieux ; " homme » = Grand Homme, se hissant au niveau de sa fonction.

Le « distinguo subtil » est fragile. À preuve les exemples d'antimétaboles qui correspondent à une réversion, si on prend en compte le critère du changement de sens : ainsi, de "Rome était dans notre camp et notre camp dans Rome » (Corneille, apud Dubois et alii 1970: 121), dans lequel "Rome » et "camp » prennent dans l'énoncé inversé une extension différente qui se superpose au sens de la première proposition. À preuve également le fait qu'en (11), si les termes ne changent ni de sens ni de référent (puisqu'il s'agit d'un NP et d'un pronom personnel), ils changent de valeur discursive avec l'inversion : «Pinguet et moi » sacrifie à la politesse, «moi et Pinguet » marque un rétablissement de la hiérarchie militaire qui convient mieux au sentiment de supériorité du caporal... Ce phénomène est accentué par le fait que l'ordre "Pinguet et moi » représente la norme : le c'est-à-dire n'indique donc pas une simple correction, mais pointe sur un effet discursif. Mutatis mutandis, il en va de même en (13) :

(13) Récit de la violence $e t$ violence $d u$ récit. 
Dans ces sortes d'énoncés - fréquents dans les titres des exposés universitaires -, la première occurrence de "récit » a pour synonyme la fabula ou l'histoire, c'est-à-dire la trame événementielle de l'intrigue, tandis que la deuxième occurrence renvoie à la narration, autrement dit à une manière de raconter. De même, "violence » n'a pas le même référent, selon qu'il renvoie à l'histoire ou à sa narration. Ces différences de sens sont bien sûr tributaires de la préposition qui hiérarchise les relations entre ces termes à l'intérieur des groupes de mots. Par conséquent, le critère de la permanence du sens est instable dans l'antimétabole, ce qui fragilise la distinction d'avec la réversion.

Cette distinction est également contestée par ceux qui définissent l'antimétabole comme la figure dans laquelle le renversement de l'ordre des mots est mis au service du renversement de l'ordre des propositions (Honeste 2007 : 403). En effet, dans

(14) Il faut vivre pour manger et non manger pour vivre.

l'antimétabole ne se limite pas à formuler deux propositions contradictoires ([vivre pour manger] vs [manger pour vivre]), mais rapporte ensemble ces dernières, afin que le coénonciateur interprète cette cooccurrence comme le renversement d'un ordre doxique au profit d'un ordre plus pertinent.

On fait l'hypothèse que la différence entre réversion et antimétabole, du fait de la fragilité de la permanence du sens des mots inversés, est secondaire par rapport aux fonctionnements syntaxiques et aux valeurs sémantiques qui distinguent d'une part les $\mathscr{A}$ - dans lesquelles l'inversion des propositions annule l'une des deux, comme en (14), d'autre part les $\mathscr{A}+$, dans lesquelles l'inversion des propositions n'annule pas une prédication au profit de la seconde, comme en (13) et en (15).

En (15) l'inscription célèbre de mai 1968 renverse l'énoncé figé à valeur proverbiale, produisant un énoncé étonnant, non dénué de pertinence, si l'on pense à la valeur métonymique des " oreilles » et à la valeur métaphorique du « mur » :

(15) Les murs ont des oreilles. Les oreilles ont des murs.

L'antimétabole renvoie à la classe de ceux qui, emmurés dans leurs certitudes, ne comprennent pas les motivations de la jeunesse ${ }^{7}$. Cette idée n'annule pas le point de vue $\left(\mathrm{PDV}^{8}\right)$ doxique de la première proposition, bien au contraire : elle établit un lien entre un conservatisme insensible à la nouveauté et un autre, basé sur la délation, renvoyant dans l'interdicours au conservatisme par excellence de la Collaboration sous Vichy.

Ces deux classes $\mathrm{d}^{\prime} \mathscr{A}$ - et + reposent sur un principe explicatif commun, à travers la prise en compte de la théorie énonciative du PDV : l'envisagement ${ }^{9}$

7. Explication qui intègre celle selon laquelle « les oreilles » sont aussi celles du Grand Charles (de Gaulle) qui représente le plus la classe dirigeante, par son rôle institutionnel.

8. Sur la notion de PDV, voir l'introduction à ce volume, ainsi que Ducrot 1984 et Rabatel 2005.

9. Nous risquons le néologisme : il est, à tout prendre, meilleur que le «mode d'apercevance des phénomènes »de Damourette et Pichon 1911-1936, t. 5 : 174). L'« envisagement» nous semble dire assez heureusement une manière dialogique de concevoir les choses, d'exprimer un PDV 
des choses selon un mode inversif n'implique pas nécessairement des PDV antagonistes exclusifs, mais repose a minima sur la coexistence de PDV complémentaires. L'antimétabole apparaît dès lors comme une figure privilégiée qui traite du même et de l'autre, selon des tensions qui la portent tantôt vers l'expression de PDV antithétiques divergents $(\mathscr{A}-)$, tantôt vers celle de PDV qui convergent au-delà de leur différences initiales $(\mathscr{A}+)$.

\section{ANALYSES SYNTAXIQUE-ÉNONCIATIVE ET SÉMANTIQUE-ÉNONCIATIVE DES ANTIMÉTABOLES}

L'accent mis sur l'énonciation, dans le titre, indique qu'à nos yeux, les relations syntaxiques et les valeurs sémantiques gagnent à être référées aux instances qui prennent en charge les contenus propositionnels. Le locuteur/ énonciateur premier (L1/E1), celui qui prend en charge les énoncés comme vrais, doit être distingué des locuteurs/énonciateurs seconds, auxquels L1/E1 impute un certain nombre de PDV, sans nécessairement les prendre en charge, se contentant de les prendre en compte, quitte à s'en distancier ou au contraire à les faire siens. Ce modèle montre que L1/E1 ne se réduit pas aux situations relativement claires des énoncés pris en charge ou récusés, mais qu'il joue aussi sur l'immense masse des situations grises dans lesquelles L1/E1 joue avec les PDV qu'il prend en compte et avec lesquels il interagit de façon dialogique pour construire son discours et y faire entendre sa propre "voix", en tant que principal (cf. ici même, l'introduction).

La difficulté de l'analyse de l'antimétabole repose sur l'intrication de deux phénomènes différents, l'inversion des mots et l'inversion des propositions, se manifestant dans des structures variées. Car il existe des inversions dans lesquelles la hiérarchisation est marquée par les rections à l'intérieur de chaque prédication (cf. (14)), mais non entre les prédications, et d'autres dans lesquelles la subordination est également marquée entre prédications, comme dans l'exemple suivant :

(16) Vivre simplement pour que d'autres puissent simplement vivre. (Gandhi)

Ces différences rejaillissent sur la distinction des $\mathscr{A}$ - et + .

\section{I. Antimétaboles MOINS : prise en charge énonciative de la proposition dominante par LI/EI, prise en compte plus ou moins distanciée de la proposition dominée}

La proposition prise en charge est celle qui, sur les plans syntaxique, sémantique et énonciatif, domine l'autre. Le critère n'est pas positionnel, ce n'est pas nécessairement la proposition inversée qui domine. C'est vrai pour les anti-

\footnotetext{
$>$ en les envisageant de telle ou telle façon, bref, de renvoyer aux diverses possibilités qu'a le locuteur d'adopter des positions énonciatives variables quant aux objets ainsi que des postures également variables par rapport aux interactants de la communication.
} 
métaboles comportant un $X$ mais $Y$, un $X$ pourtant $Y$ (voir (17)), mais cette hiérarchisation tient moins à l'ordre inversif qu'à la valeur du marqueur : à preuve le fait que les antimétaboles comprenant un marqueur du type plutôt X que $Y, X$ et non $Y, X$ pour que $Y$ sélectionnent $X$ en position dominante, comme en (18)(19), qui sont semblables à (16) :

(17) Pendant des décennies, au Brésil on disait : « il faut croître pour distribuer. » Mais nous, nous distribuons pour croître. (Lula, Le Monde 25-5-2006)

(18) Plutôt la retraite à la maison que la maison de retraite. (de Villiers, Campagne officielle, 18-4-2007)

(19) Il faut manger pour vivre et non vivre pour manger.

La position dominante d'un PDV peut également être indiquée par le sémantisme d'un verbe, comme «je préfère... que », qui relève de la structure plutôt $X$ que $Y$, dans (20), ou par la place de la principale après relative antéposée de (21) :

(20) Comme je l'ai déjà dit avec un peu d'humour, je préfere dire : "voici, c'est mon programme », que «mon programme c'est Voici. » (L. Fabius, France Inter, 27-8-2006)

(21) Vous qui avez accouché sans coucher/pécher ${ }^{10}$, laissez-nous coucher/ pécher sans accoucher. (Prière à la Vierge des prostituées anversoises)

Bref, nous sommes ici face à un premier type d'antimétabole dans lequel l'inversion des mots et des prédications infirme la validité d'une des prédications au profit de la prédication dominante, qui est seule prise en charge comme vraie par L1/E1, tandis que la prédication rejetée ou mise à distance n'est que prise en compte comme indice d'un PDV erroné ou doxique, en tout état de cause d'un PDV pris en compte, concédé, mais pas pris en charge : ainsi, en (21), les prostituées peuvent très bien accepter le PDV doxique sur la Vierge, mais elles ne le revendiquent pas pour elles... L'invalidation (20), ou, à tout le moins, la non-prise en charge (21) d'une des prédications, quelle que soit sa place, correspond au fonctionnement sémantico-syntaxique des $\mathscr{A}$-, et il est indiqué par un marqueur de hiérarchisation en position inter-propositionnelle. La preuve de ces différences entre prédications prise en charge et prise en compte est que l'interversion des prédications entraîne du même coup une inversion des rapports hiérarchiques entre $X$ et $Y$. Ainsi l'interversion des énoncés ne peut que renvoyer à un adversaire politique de Lula en (17'), à un adversaire des vieux et de la Famille, en (18') :

(17') Pendant des décennies, au Brésil on disait: «il faut distribuer pour croître. » Mais nous, nous croissons pour distribuer.

$\left(18^{\prime}\right) \quad$ Plutôt la maison de retraite que la retraite à la maison.

Bref, dans ce cas, L1/E1 ne prend plus en charge les mêmes prédications, ce qui revient à dire que ce n'est plus le même PDV qu'il prend à son compte en tant que locuteur/énonciateur principal.

10. Exemple fourni par le guide qui fit la visite d'Anvers lors du colloque Prise en charge énonciative (2007). La version avec " coucher » est meilleure que celle avec "pécher", moins parce que l'idée du péché est absente, que parce que le jeu des sonorités avec accoucher est plus subtil... 
En l'absence de marqueur de subordination, le PDV dominant est marqué au niveau intra-propositionnel, par des connecteurs, des prépositions ou la valeur argumentative intrinsèque du lexique. Dans ce cas, l'ordre des propositions n'a pas d'influence sur la signification, qui reste la même dans tous les cas, que les propositions soient juxtaposées ou coordonnées :

(22) Vivre simplement ou simplement vivre?

(22') Simplement vivre ou vivre simplement?

(23) Manger pour vivre ou vivre pour manger?

(23') Vivre pour manger ou manger pour vivre?

L'interversion des prédications, en $(22) /\left(22^{\prime}\right)$ et en $\left(23 /\left(23^{\prime}\right)\right.$, n'affecte pas la hiérarchisation interne des deux prédications: il est toujours préférable de " manger pour vivre", de "vivre simplement », quelle que soit la place de ces prédications, tandis que les autres termes de l'alternative sont orientés négativement, dans un cas par l'antéposition de l'adverbe, dans l'autre par l'activité de manger, peu valorisée du fait de la préposition, qui envisage l'activité comme si elle occupait tout l'espace vital. Dans les deux cas, il n'y a pas de véritable interrogation, mais une affirmation qui prend la forme d'une question rhétorique. La hiérarchisation est un fait de syntaxe et de lexique; elle est suffisamment forte pour être indifférente à l'interversion des propositions coordonnées ou juxtaposées. Autrement dit, dans tous les cas, un seul des deux PDV est pris en charge par L1/E1, l'autre, attribué à un énonciateur second, n'est que pris en compte ${ }^{11}$.

\subsection{Antimétaboles PLUS : double prise en charge des deux propositions}

Les $\mathscr{A}+$ sont celles qui reposent sur une double prise en charge par L1/E1 des deux prédications. Il s'ensuit que l'interversion de l'ordre des propositions n'influe pas sur le sens global des prédications ni des prises en charge. Ces $\mathscr{A}+$, telles celles de (24) et (25), acceptent que l'inversion de l'ordre des propositions produise des significations certes différentes, mais au total complémentaires, parce que les divergences sont moins fortes que les convergences. Ce phénomène est bien évidemment davantage marqué avec les coordinations par et mais il fonctionne aussi avec des énoncés juxtaposés (cf. (24") et (25") :

(24) Désormais, chère Ségolène, c'est une pour tous, et tous pour une! (L. Fabius, Grand-Quevilly, 23-2-2007, premier meeting réunissant Fabius et Royal)

(25) Tristan Bernard avait senti le danger : «Quand on bloque les comptes $e t$ que l'on compte les Bloch, il faut s'inquiéter. ${ }^{12} \gg C^{\prime}$ 'était sous l'occupation. (L. Joffrin, Libération, 23-2-2007 à propos du débat sur l'instauration de statistiques raciales)

11. Sur les différences entre prise en charge et prise en compte, voir le numéro de Langue française (à par.) consacré à la prise en charge.

12. (25) est un cas limite d'antimétabole car il repose sur un jeu de mots. Néanmoins, la réalité des mécanismes inversifs (plus acceptable à l'oral qu'à l'écrit) justifie son intégration dans notre corpus. 
(24') Tous pour une et une pour tous.

$\left(24^{\prime \prime}\right)$ Tous pour une, une pour tous.

(25') Quand on compte les Bloch et que l'on bloque les comptes.

$\left(25^{\prime \prime}\right)$ Quand on compte les Bloch, bloque les comptes.

Le sens des énoncés (24), (24'), (25), (25’) est le même, indiquant la solidarité de phénomènes distincts, mais subsumés par l'idée d'unité dans le premier cas, de dérive raciale dans le deuxième. La répétition et l'inversion n'enlèvent rien, elles renforcent par le dire un dit jugé équivalent, tout en prenant acte que l'équivalence du dit est enrichie par ces envisagements successifs. Dans les $\mathscr{A}+$, la deuxième proposition vient s'ajouter à la première en indiquant une addition et/ou une gradation, ou une rectification. Dans les deux cas, la première proposition n'est pas annulée, elle est précisée, complétée : à preuve la possibilité d'incrémenter entre les deux un et, un voire, un et en plus (addition, gradation), ou un autrement dit, un ou (rectification) ${ }^{13}$. Ainsi le et de (26) (et, plus loin, de (30)) exprime une addition de deux prédications, voire une addition avec gradation (et = et en plus), ce qui est bien sûr la meilleure preuve de la double prise en charge :

(26) Désormais, chère Ségolène, c'est une pour tous, et en plus tous pour une!

Les deux prédications coordonnées par un et indiquent une prise en compte de PDV complémentaires qui ne le sont pas a priori : c'est ce qu'indique le désormais de (26), trace de tensions entre les candidats à la candidature. Il en va de même, en (13): le et indique, au-delà des différences de hiérarchisation marquées par les rections, une osmose entre un univers thématique violent et un mode d'expression violent, témoignant, par-delà la discordance, d'une convergence dominante.

L'A + exprime la complémentarité de deux points de vue distincts et complémentaires, dont le prix est précisément de rassembler ce qui était à première vue opposé, sans nécessairement annuler l'une des deux prédications. Bref, il s'agit de montrer, au sens linguistique du terme, qu'aucune des analyses n'épuise la complexité du phénomène, ni la thèse de l'opposition radicale, ni celle de la ressemblance, puisque la figure montre un parcours, de l'un vers l'autre (26), du même vers l'autre, et de l'autre vers le même (8).

Ce type d'indifférence à l'ordre des prédications se rencontre dans d'autres cas, par exemple dans des prédications inversives coordonnées par un ou :

(27) Alors, belle époque pour l'art médiocre ou médiocre époque pour le bel art?» (Arte, Métropolis, 7-4-2007)

$\left(27^{\prime}\right)$ Alors, médiocre époque pour le bel art ou belle époque pour l'art médiocre?

En $(27) /\left(27^{\prime}\right)$, 1'ordre des propositions est indifférent à la conclusion, parce qu'en définitive les deux réponses sont subsumables par l'idée de décadence, même si, dans un cas, c'est l'art qui est décadent, dans l'autre l'époque. On pourrait aussi alléguer la présence de la forme interrogative, mais il semble que

13. La possibilité d'incrémenter des marqueurs de la convergence dans les $\mathscr{A}+$ et dans les fausses antimétaboles pourrait plaider en faveur de leur rapprochement. Mais ce serait oublier qu'il existe deux PDV dans les $\mathscr{A}+$, et un seul dans les fausses antimétaboles. 
derrière l'apparente libéralité de l'ordre des propositions et donc des PDV, il faille interpréter ces énoncés en un sens relativement contraint par une doxa valable quel que soit l'ordre des prédications, les énoncés exprimant la même conception du monde ${ }^{14}$. Ce raisonnement peut être étayé par le fait que la question appelle plutôt comme réponse : " $X$ est vrai, mais $Y$ aussi » ou « $X$ et $Y$ sont vrais à des degrés variables ». Si l'on ajoute la possibilité d'incrémenter un adverbe tel indifféremment, on comprend qu'en effet, la vérité des propositions ne tient pas à leur ordre. Ce phénomène se rencontre aussi à la forme affirmative :

(28) Bela Bartok, le plus moderne des classiques, sinon le plus classique des modernes. (Télérama, 16-9-1995)

$\left(28^{\prime}\right)$ Bela Bartok, le plus classique des modernes, sinon le plus moderne des classiques.

En (28)/(28'), l'interversion des propositions étant sans effet, $\left(28^{\prime}\right)$ est une paraphrase acceptable de (28), parce que les deux prédications sont subsumées par la thèse que, pour les grands artistes, l'opposition entre classicisme et modernité n'est pas pertinente, les grands génies étant à la fois innovants (modernes) et intemporels (classiques) : c'est d'ailleurs bien le sens du sinon indiquant une rectification qui, puissantiellement, porte en elle la réversibilité de la double prédication ${ }^{15}$. Bref, formulés interrogativement, $(28)$ et $\left(28^{\prime}\right)$ admettent comme réponse la plus probable que $X$ et $Y$ sont vrais, ou, plus exactement, qu'ils recèlent une partie de la vérité.

Dans cette deuxième classe d'antimétabole, $\mathscr{A}+$, les deux prédications sont prises en charge comme vraies par L1/E1, indépendamment de leur interversion, parce qu'elles n'opposent pas deux PDV contradictoires, mais exhibent deux PDV complémentaires, ce que confirment les incrémentations qui renforcent la co-orientation des énoncés ${ }^{16}$.

En définitive, privilégier une de ces $\mathscr{A}+$ ou - au détriment de $1^{\prime}$ autre ${ }^{17}$, ce serait ne voir qu'une des facettes de la dualité d'une figure faite précisément pour penser la complexité.

14. Le contexte est ici déterminant : un tel énoncé en ouverture ou en clôture n'a pas forcément la même orientation.

15. Cette explication repose sur l'idée que sinon ne joue pas vraiment un rôle de réfutation d'une des prédications (auquel cas on serait face à une $\mathscr{A}-$, comme le remarque B. Verine), mais au contraire indique une relativisation du dit et/ou du dire : en ce sens, la concession exprimée par sinon est l'équivalent de à moins que ce ne soit (modalisation du dit) ou à moins qu'il ne faille dire (modalisation du dire).

16. Il y aurait à approfondir l'investigation de la différenciation des marqueurs dialogiques selon la classe d'antimétabole : sans doute trouvera-t-on dans l' $\mathscr{A}$ - (comme dans le chiasme et l'antithèse) davantage de marqueurs de négation, d'opposition, de concession et dans les $\mathscr{A}+$ davantage de marqueurs de convergence, de co-orientation : et, en plus, voire, sinon, disons, autrement dit, c'est-àdire; de même, dans le premier cas, les particules intensives, la nomination, le jeu des valeurs aspectuo-temporelles, etc. s'appuieront sur la divergence, dans le second sur la convergence.

17. Frédéric 1985: 183s ne retient que la convergence («Figure suprasegmentale marquant la convergence»). Honeste 2007 : 403 privilégie la divergence, du moins dans son résumé de communication pour le colloque du CILPR 2007. 


\section{ANALYSE PRAGMATIQUE DE LA FIGURE DE L'ÉNONCIATEUR DE FIGURES INVERSIVES : COMPLEXITÉ DES CHOSES, ESPRIT DE CONTRADICTION, ETHOS DU REDRESSEUR DES IDÉES FAUSSES}

\section{I. Figures inversives et opacification des dires/des dits}

Les figures inversives alimentent l'image discursive (ou figure ${ }^{18}$ ) d'un locuteur/énonciateur maître de sa parole et de son vouloir dire, doté d'une compétence langagière hors norme (Forget 2000: 169, Berrendonner 2001). Si la maîtrise des figures est l'indice d'une expertise linguistique, compte tenu de la complexité des mécanismes inversifs à l'œuvre, il n'en reste pas moins qu'il serait erroné d'interpréter la dynamique inversive uniquement en termes de maîtrise et de clarté :

l'usage des figures ne doit pas s'interpréter ici comme beau langage, joliesse : il s'agit d'accepter que la langue opacifie, qu'il se crée du fait de la langue des relations auxquelles le locuteur n'a pas nécessairement pensé ou auxquelles il ne voudrait pas accorder de lui-même un crédit important, etc. (Barthes, 1973, 2002c : 448)

Barthes relie ce processus d'opacification avec le phénomène de la signifiance (Barthes 1973, 2002 : 450), caractéristique des textes littéraires, opposant ainsi l'activité des écrivains qui acceptent «de renoncer aux garanties de la rédaction transparente, instrumentale » à celle des scripteurs (écrivants) qui se servent de la langue comme d'un outil de communication transparent (Barthes 1970, 2002 : 671). Toutefois, l'opposition entre opacification et transparence ne se limite pas à cette dichotomie, ainsi que l'a montré Authier-Revuz à propos de l'opacification des boucles méta-réflexives: les tensions entre transparence et opacification sont constitutives du jeu langagier, dans sa réflexivité tous azimuts, pour penser les référents, les mots, les situations.

Certes, les antimétaboles, qui jouent avec l'interdiscours, sont a priori plutôt mémorisables en raison de leurs caractéristiques formulaires, et paraissent pour cela de bonnes candidates pour devenir des énoncés mémorables, mais la complexité de la structure, et les phénomènes d'opacification qui l'accompagnent, n'en facilitent pas la production et la reproductibilité. L'antimétabole fait partie de ces figures auxquelles on réfléchit mûrement pour les formuler. Mais passé l'effet de sidération, et le crédit porté à la performance du locuteur, dès que l'on se met à réfléchir, les choses sont toujours plus opaques que ce que semblent en dire ces renversements péremptoires...

(29) Vous savez, celui qui a le sens de la formule qui ne formule pas beaucoup de sens. (un partisan de «Désir d'avenir » contre N. Sarkozy, Antenne 2, 10-12-2006)

18. La figure discursive de l'énonciateur englobe l'ethos, mais ne s'y réduit pas, puisqu'elle est également construite par un certain usage du logos et du pathos. 
(30) Mais je ne suis pas un conservateur, car je crois au mouvement. L'ordre n'est acceptable que s'il est en mouvement. L'ordre juste, c'est juste l'ordre. (N. Sarkozy, Le Progrès, 30-11-2006)

La formule est percutante, mais elle reste courte, car l' « ordre juste », même si l'on trouve la formule discutable ${ }^{19}$, mérite plus d'attention à la notion de justice, ici évacuée. De même, la formule finale de F. Hollande paraît-elle définitive, mais, à y réfléchir, elle est la trace d'une difficulté politique et d'une opacification qui repose sur le coup de force autour du marqueur de reformulation à la charnière des deux propositions inversées :

(31) Au milieu [entre les propositions de Strauss-Kahn et Fabius], François Hollande ménage la chèvre et le chou: "Si d'autres, au second tour de l'élection, veulent venir avec nous pour battre la droite et mettre en œuvre le pacte, ils seront les bienvenus, expose-t-il dans un entretien à Libération, lundi. Mais ce n'est pas la démarche de François Bayrou. Lui, veut une alliance des contraires, c'est-à-dire le contraire d'une alliance ». Le PS a un problème. Que faire de François Bayrou. (Le Monde, 13-3-2007)

En (31), le marqueur de reformulation paraphrastique $c^{\prime} e s t-\grave{a}$-dire est paraphrasable par autrement dit, en fait, indiquant une reformulation qui dévoile les non-coïncidences entre les mots et les choses, et donc le marché de dupes de l'ouverture au centre. F. Hollande fait comme si l'opposition gauche/droite allait de soi, ce qui est loin d'être le cas pour les partisans - dont la candidate socialiste - d'une alliance du PS avec le centre. C'est pourquoi, quel que soit le brio de sa formule, Hollande est dans une situation difficile, que le journaliste rappelle non sans cruauté...

\subsection{Tensions entre figures d'énonciateur de la complexité des choses et figures d'énonciateur du polémiste anticonsensuel}

La fonction argumentative des figures inversives joue sur le logos, mais aussi sur l'ethos et le pathos. Si discours des passions il y a, il est convoqué sur le dos des personnes, notions ou événements qui font l'objet d'une structure inversive, en complicité avec un coénonciateur complice qui partage l'ethos de celui qui veut avoir le dernier mot, comme en (30). Complicité et connivence s'exercent aussi à l'égard des énonciateurs internes à l'antimétabole que L1/E1 met à distance, comme on le voit en (18) : car celui qui est présenté comme le partisan de la retraite dans des lieux organisés pour soigner les dépendances (à l'instar de la maison de retraite, terme bien inapproprié et daté pour évoquer de tels lieux) est de ce fait présenté du même coup un adversaire de la famille, ainsi que L1/E1 le donne à penser.

Il s'ensuit que la fonction cognitive de l'antimétabole (et du chiasme) est subordonnée à une fonction informative et argumentative forte ${ }^{20}$, influant sur l'ethos de l'énonciateur. Ce dernier se situe dans un cadre qui, sans être néces-

19. Voir les analyses de Taguieff, $2007: 54$ et plus largement 51-66.

20. «La fonction cognitive de la figure, en dépit de ses profondes parentés avec la démarche scientifique, en diffère sur certains points : le savoir scientifique fait abstraction des situations, 
sairement polémique, repose a minima sur le refus de se satisfaire des manières traditionnelles de voir : c'est pourquoi les inversions peuvent souvent s'accompagner des opérations de mises en relief, de focalisation et de concrétisation ${ }^{21}$. La fonction cognitive est exhibée, elle dit/montre les contradictions ou la complémentarité des PDV sur un même référent. L'énonciateur de ces figures adopte une posture énonciative de dévoilement, traversée, éventuellement, par un devoir d'irrespect, au nom d'une vérité supérieure, en mettant les rieurs de son côté, et, en définitive, en tentant d'imposer comme seul pertinent le PDV de l'énonciateur figural : ce type de positionnement énonciatif est compatible avec $l^{\prime} \mathscr{A}-$, mais il $l^{\prime}$ est aussi avec $l^{\prime} \mathscr{A}+$, qui insiste sur la complexité des choses et sur la nécessité de multiplier les PDV pour en rendre compte sans exclure de PDV, et sans se livrer à des polémiques. Toutefois, la limite entre ces deux figures d'énonciateur est poreuse, comme le montrent ces exemples, qui affichent une postulation de complexité et discréditent celui qui s'avère incapable de la saisir, a fortiori de la penser :

«En fait, ce marxisme est mythique puisque, lorsqu'il réfléchit sur la question juive, Marx n'est pas marxiste, et lorsqu'il est marxiste il ne réfléchit pas sur la question juive » [Misrahi, Marx et la question juive, Gallimard, 1972 : 90]. De même, quand le marxisme réfléchit sur l'écriture, il n'est pas marxiste, et quand il est marxiste, il ne réfléchit pas sur l'écriture. (Meschonnic, Pour la poétique 2, 1973 : 296)

(33) À l'encontre de la philosophie allemande qui descend du ciel sur la terre, c'est de la terre au ciel que l'on monte ici. Autrement dit, on ne part pas de ce que les hommes disent, s'imaginent, se représentent, ni non plus de ce qu'ils sont dans les paroles, la pensée, l'imagination et la représentation d'autrui, pour aboutir ensuite aux hommes en chair et en os ; non, on part des hommes dans leur activité réelle. [...] Ce n'est pas la conscience qui détermine la vie, mais la vie qui détermine la conscience. » (Marx et Engels, L'idéologie allemande, apud Lahire 2006 : 20)

C'est pourquoi la figure du polémiste n'est jamais loin. À la différence de l'euphémisme, qui donne du référent une image aseptisée et dédramatisée, en lien avec une polyphonie convergente du on-dit diluant les responsabilités, reposant sur un effacement de l'engagement discursif des locuteurs/énonciateurs (Bonhomme 2005 : 243-247), $1^{\prime} \mathscr{A}-$, mais aussi le chiasme et l'antithèse, reposent sur une image non consensuelle du locuteur, sur un refus de l'irénisme, sur une conception éristique et agonistique du discours. Il y a de l'ethos

du locuteur, des récepteurs, prix de sa prévisibilité et falsifiabilité ; la figure repose sur une appréhension phénoménologique individualisée » (Klinkenberg 2000 : 83). En outre, l'interprétation scientifique des énoncés repose sur une signification exclusive, l'interprétation rhétorique est cumulative, parce que les unités d'un énoncé peuvent avoir plusieurs sens ou l'énoncé plusieurs lectures (ibid. : 86). L'aptitude au cumul permet à la figure de jouer un rôle cognitif original, en résolvant des contradictions, en expérimentant, en proposant des médiations entre les termes disjoints de ces problèmes ou de ces contradictions (ibid. : 72, 75-80). Ce phénomène est à l'œuvre dans les figures qui modifient les relations entre concepts ou assignent de nouvelles catégorisations à des concepts, telles la métaphore ou de l'antimétabole.

21. Qui jouent un rôle important dans la fonction cognitive des figures (Bonhomme 2005 : 172-174). 
du pamphlétaire (Angenot 1982) dans $1^{\prime} \mathscr{A}$-, un même refus de la pondération/ modération, un même refus des opinions convenues et de la langue de bois, à ceci près qu'ici, il s'agit d'un refus d'opinions doxiques qui s'identifient à des formules repérables dans l'interdiscours, mais dont la visibilité est limitée dans le temps ou dans l'espace, et qui ne relèvent pas des langues de bois.

Toutefois, nos observations antérieures invitent à ne pas ratifier l'idée que la figure de l'énonciateur de figures inversives se poserait en extériorité radicale face aux autres ou à la doxa, ni que $1^{\prime} \mathscr{A}$ - serait l'essence de la figure. Au contraire, la manière de citer l'autre, de s'en distancier en en gardant, fussent-ils réorganisés autrement, les éléments qui servent de cadre à une nouvelle prédication, montre que, le plus souvent, le même et l'autre sont fortement intriqués.

$C^{\prime}$ est là le rôle inappréciable de $1^{\prime} \mathscr{A}+$, qui repose sur un ethos moins guerrier, et qui met davantage en avant la complexité épistémique - ce qui n'empêche pas les conflits qui jalonnent la vie scientifique. Ainsi, les parcours envisagés dans les $\mathscr{A}+$ construisent-ils diverses figures de l'énonciateur, selon les incrémentations qui explicitent le sens du parcours. De... à signifie la volonté d'exhaustivité, invitant à faire le tour de la question (d'où son succès dans la prose universitaire) :

(34) De la violence de l'écriture à l'écriture de la violence.

(34') De l'écriture de la violence à la violence de l'écriture.

Répétés dans un ordre inverse, (34) et (34') construisent une figure d'énonciateur exhaustif aisément partageable par le coénonciateur parce que la nécessité d'envisager les deux parcours et leurs points de vue complémentaires découle moins d'un caprice du locuteur que de la complexité même des choses ou du langage ${ }^{22}$, selon une représentation mimologique ${ }^{23}$ qui a cours en dépit de son caractère pré-scientifique, comme si les mots, leur répétition dans un ordre inverse signifiait quasi magiquement l'existence d'une complexité.

L'incrémentation d'un voire construit un autre ethos : comme si l'énonciateur envisageait ce retournement par scrupule d'exhaustivité tout en étant conscient des effets de mode de ces manières de dire. Dans ce cas là, voire s'accompagne d'une mimique et d'une intonation de distanciation, éventuellement explicitées par un si j'ose dire, je dirais même, comme on dit, ou par toute autre formule par laquelle le locuteur modalise son dire et souligne des formes de non-coïncidences :

Violence de l'écriture, voire, si j'ose dire, écriture de la violence.

Écriture de la violence voire, si j'ose dire, violence de l'écriture.

22. Sur ce plan, l'antimétabole se rapproche du paradoxe verbal : voir Landheer, à paraître.

23. Cf. Genette 1976 et Barthes (1972/2002b : 32s) : «L'antithèse n'est pas seulement une figure emphatique, c'est-à-dire en somme un simple décor de la pensée ; c'est probablement autre chose et plus; une façon de faire surgir le sens d'une opposition de termes; et comme nous savons par les explorations de la linguistique que c'est là le procédé fondamental de la signification (et certains physiologistes disent même de la perception), nous comprenons mieux que l'antithèse [...] est devenue une pointe, c'est-à-dire le spectacle même du sens. » 
Résumons les points saillants de ce parcours :

i : La différence sémantique entre antimétabole et réversion n'est pas solide, d'abord parce que le référent varie le plus souvent, ensuite parce que même dans le cas le plus favorable à la différence des deux figures où le référent reste le même, les différences d'envisagement en modifient les valeurs.

ii : La notion de hiérarchisation syntactico-sémantique est fondamentale : elle opère soit à l'intérieur des groupes de mots, soit entre les propositions, soit dans les groupes de mots et entre les propositions.

iii : La saisie des PDV en confrontation dans les propositions inversées dégage deux fonctionnements de l'antimétabole : dans $l^{\prime} \mathscr{A}+$, la sélection du PDV dominant n'empêche pas le cumul des deux PDV pris en charge par L1/ E1, tandis que dans $l^{\prime} \mathscr{A}$-, cette même sélection implique un rejet ou une distanciation du PDV pris en compte au profit du seul PDV pris en charge par L1/E1.

iv : $\mathrm{L}^{\prime} \mathscr{A}$ - ne se réduit pas à une confrontation de PDV agoniques sur le plan interactionnel et $1^{\prime} \mathscr{A}+$ à une confrontation cognitive pacifique de PDV qui chercherait à dire la complexité par le dépassement des PDV particuliers. Le conflit, comme la convergence, concernent possiblement et les interactions et les confrontations notionnelles.

$\mathrm{v}$ : Les $\mathscr{A}+$ et - alimentent des figures d'énonciateur consensuelles et/ou agoniques (avec des degrés) dénotant une réelle maîtrise linguistique. Mais celle-ci est relativisée d'abord par le nombre d'antimétaboles comportant des forçages, des à-peu-près - cf. (3), (18), (20), (25) -, ensuite par les mécanismes d'opacification qui la sapent de l'intérieur.

Nous n'avons pas développé l'analyse des fonctions pragmatiques des figures inversives en privilégiant une scène d'énonciation spécifique, en l'occurrence celle du monde politico-médiatique. Gageons que, resituées dans leur co-texte, les figures apparaîtraient comme l'acmé d'un processus figural plus global : les antimétaboles, comme d'autres figures inversives, sont tantôt un point de culmination qui conclut un réseau dense d'oppositions, tantôt - surtout en contexte dialogal - l'amorce brillante d'une idée développée ultérieurement. Il s'ensuit que la figure de l'énonciateur figural ne se réduit pas à la figure, elle est construite par l'ensemble de la dynamique discursive et interactionnelle, comme on l'entrevoit en (31), (32), (33). Nous n'avons pas davantage exploré la problématique des postures énonciatives (Rabatel 2004), même si l'on peut faire l'hypothèse que $1^{\prime} \mathscr{A}$ - repose sur un mécanisme de surénonciation - l'énonciateur principal ne prenant en charge que le PDV inversé, qui déprécie de facto le contenu du PDV dominé pris en compte - tandis que $1^{\prime} \mathscr{A}+$ relève plutôt de la coénonciation, avec une double prise en charge des PDV. Ce sont là des données qui restent à investiguer, d'autant plus sûrement, on l'espère, que le socle de l'antimétabole est désormais plus solide. 


\section{Références bibliographiques}

ANGENOT M. (1982) La parole pamphlétaire. Payot, Paris.

AQUIEN M. (1993) Dictionnaire de poétique. Le Livre de Poche, Paris.

BARTHES R. (2002a) [1970] «L'Express va plus loin avec... Roland Barthes », in Fuvres complètes t. 3, 1968-197|, 67|-688. Éditions du Seuil, Paris.

BARTHES R. (2002b) [1972] «La Rochefoucauld: Réflexions ou Sentences et maximes », in Fuvres complètes t. 4, 1972-1976, 25-40. Éditions du Seuil, Paris.

BARTHES R. (2002c) [1973] «Texte (théorie du) 》, Euvres complètes t. 4, 1972-1976, 443-459. Éditions du Seuil, Paris.

BerRendonner A. (200 I) «Portrait de l'énonciateur en faux naiff 》, Semen I5, I I3- I 25.

Bonhomme M. (2005) Pragmatique des figures du discours. Champion, Paris.

DAMOURETTE et PICHON (1911-1936) Des mots à la pensée. Essai de grammaire de la langue française. Tome cinquième. D'Artrey, Paris.

Dubois J., Edeline F., Klinkenberg J.-M., Minguet P., Pire F., Trinon H. (1970) Rhétorique générale. Larousse, Paris

DUCROT O. 1984 Le dire et le dit. Éditions de Minuit, Paris.

DUPRIEZ B. 1980 [1977] Gradus. Les procédés littéraires. 10/18, Paris.

FONTANIER P. 1968 [I82I] Les figures du discours. Flammarion, Paris.

FORGET D. (2000) Figures de pensée, figures de discours. Nota Bene, Québec.

FRÉDÉRIC M. (1985) La répétition. Étude linguistique et rhétorique. Max Niemeyer Verlag, Tübingen.

Genette G. (1976) Mimologiques. Éditions du Seuil, Paris.

HONESTE M.-L. (2007) «Subversion de la syntaxe et syntaxe de la subversion », Communications: Résumés. XXVe congrès international de linguistique et de philologie romanes. Innsbruck university press, Innsbruck.

KLINKENBERG J.-M. (2000) «L'argumentation dans la figure », Cahiers de praxématique 35, 59-86.

LANDHEER R. (2007) «L'énoncé paradoxal et son parcours interprétatif: une ambivalence bien balancée », Actes du colloque Le paradoxe et ses usages. Pécs, mars 2007 (à paraître).

LAUSBERG H. (1990) [1960] Handbuch der literarischen Rhetorik. Franz Steiner Verlag, Stuttgart.

MOLINIÉ G. (1992) Dictionnaire de rhétorique. Le Livre de Poche, Paris.

MORIER H. (1998) [196I] Dictionnaire de poétique et de rhétorique. Presses universitaires de France, Paris.

POUGeOISE M. (200I) Dictionnaire de rhétorique. Armand Colin, Paris.

PREMINGER A. (éd) (1965) Princeton encyclopedia of poetry and poetics. Princeton university press, Princeton, New Jersey.

RABATEL A. (2004) «Stratégies d'effacement énonciatif et surénonciation dans Le dictionnaire philosophique de Comte-Sponville », Langages, 156, I8-33.

RABATEL A. (2005) «Le point de vue, une catégorie transversale », Le Français aujourd'hui I5I, 57-68.

RABATEL A. «Prise en charge et imputation, ou la prise en charge à responsabilité limitée... », Langue française (à par.).

REBOUL O. (1991) Introduction à la rhétorique. Presses universitaires de France, Paris.

ROBRIEUX J.-J. (1993) Éléments de rhétorique et d'argumentation. Dunod, Paris.

TAGUIEFF P.-A. (2007, 2002) L'Illusion populiste. Flammarion, Paris.

UEDING G. (éd) (1994) Historisches Wörterbuch der Rhetorik. Max Niemeyer Verlag, Tübingen. 